\title{
Aspects of non-uniqueness:
}

\section{how one source affects the reconstruction of another}

\author{
Hasson, R. and Swithenby, S.J. \\ The Open University, Milton Keynes, UK.
}

\section{Introduction}

This paper discusses one of the issues that is at the heart of biomagnetism: the non-uniqueness of the inverse problem. Using a Bayesian statistical approach general reasoning is employed to place restrictions on the fidelity of any source reconstruction algorithm. This paper continues the theoretical development started in [1] and continued in [2] to extract probabilistic information about the biomagnetic inverse problem. The biomagnetic inverse problem is to reconstruct a current density, $\vec{j}(\vec{r})$, inside a volume $Q$ (called the 'source space'). The geometry of the sensors and of the head are represented in the lead field distributions $\vec{L}_{k}(\vec{r})$ which relate current distributions to measurements by

$$
m_{k}=\int_{Q} \vec{L}_{k}(\vec{r}) \cdot \vec{j}(\vec{r}) \mathrm{d} \vec{r}
$$

where $m_{k}$ is the value of the $k$ th sensor channel measuring the current distribution $\vec{j}(\vec{r})$. In this paper we are going to investigate the uncertainty of current density predictions that are intrinsic to the measurement geometry. Other types of uncertainty arise because of noise in the sensors and external noise sources, but this has been modelled before [3].

The limitations of the measurement geometry can be investigated in two stages. The first stage is to say, "Where are the sources that can't be reconstructed?" (i.e. the 'blind spots') and a method for investigating this was presented at the Vienna conference [2]. The question investigated in this paper is, "If this is the predicted current density, what other sources may exist that cannot be reconstructed?". A typical situation where this information might be useful is where two areas of localized activity are postulated and the output of a current density algorithm shows only one area of activity. We need to know whether the measurement instrument is capable of reconstructing the second source in the presence of the dominant source.

\section{Method}

The method relies heavily on the Bayesian formulation of the problem contained in [1]. In this paper there are two adjustable parameters, namely a weighting distribution $w(\vec{r})$ defined on the source space $Q$ (which is a consistent way of inputing prior knowledge into the algorithm) and a positive number $\zeta$ (which reflects prior knowledge of the signal to noise ratio). In [1] a probability measure on the space of all possible current distributions is derived. In this paper we will use just one of the outcomes from this analysis, the covariance of two current density distributions. The formula for the covariance is:

$$
\operatorname{cov}\left(\vec{j}, \vec{j}^{\prime}\right)=\zeta X^{\mathrm{T}}(P+\zeta I)^{-1} P^{-1} Y+\frac{1}{\zeta}\left[\gamma-X^{\mathrm{T}} P^{-1} Y\right]
$$

where $P_{i j}$ is the Gramm-Schmidt matrix and is defined by

$$
P_{i j}=\int_{Q} \vec{L}_{i}(\vec{r}) \cdot \vec{L}_{j}(\vec{r}) w(\vec{r}) \mathrm{d} \vec{r}
$$

and $X_{i}, Y_{i}, \gamma$ are the overlap integrals defined by:

$$
X_{i}=\int_{Q} \vec{j}(\vec{r}) \cdot \vec{L}_{i}(\vec{r}) w(\vec{r}) \mathrm{d} \vec{r}, Y_{i}=\int_{Q} \vec{j}^{\prime}(\vec{r}) \cdot \vec{L}_{i}(\vec{r}) w(\vec{r}) \mathrm{d} \vec{r}, \gamma=\int_{Q} \vec{j}(\vec{r}) \cdot \vec{j}^{\prime}(\vec{r}) w(\vec{r}) \mathrm{d} \vec{r}
$$

The following method uses this formula to produce easily interpreted images. 
1. Fix $\vec{j}(\vec{r})$ to be either a theoretical dipole source or a computed current density image from an experiment.

2. Pick a position $\vec{r}^{\prime}$ in the source space $Q$.

3. Let $\vec{j}^{\prime}(\vec{r})=\hat{e}_{x} \delta\left(\vec{r}-\vec{r}^{\prime}\right)$ where $\hat{e}_{x}$ is a unit vector and $\delta()$ is the Dirac delta function and compute $c_{x}=\operatorname{cov}\left(\vec{j}, \vec{j}^{\prime}\right)$

4. Repeat step 3 for the other two orthogonal unit vectors $\hat{e}_{y}, \hat{e}_{z}$ to get $c_{y}$ and $c_{z}$.

5. Plot $\sqrt{c_{x}^{2}+c_{y}^{2}+c_{z}^{2}}$ at the point $\vec{r}^{\prime}$.

6. Repeat steps $2-5$ for different $\vec{r}^{\prime}$ until the source space is adequately sampled.

The left hand diagram in Fig. 1 shows the measurement geometry for the theoretical studies. It shows the source space plane $(z=0.06 \mathrm{~m})$ below a flat hexagonal geometry 37 channel system (2nd order gradiometers, baseline $5 \mathrm{~cm}$ ) in the plane $z=0.11 \mathrm{~m}$. The head is modelled as a conducting sphere centered at the origin.

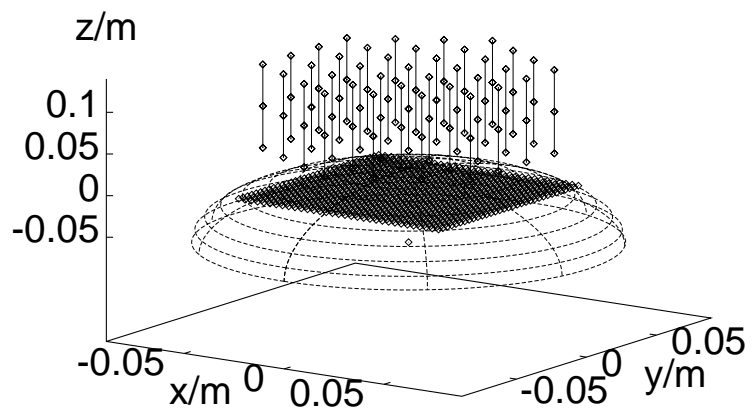

Fig. 1: (left) Measurement geometry for theoretical investigations.

\section{Examples and Discussion}

To find the 'blind spots' in the source reconstruction we must compute the overlap in the space of all possible source distributions, between the representation of the source present and the representations of all other possible sources. The Bayesian method described in [1] gives us a method of doing exactly this. In [1] a posterior probability distribution is derived on the infinite dimensional Hilbert space of all possible current density distributions. Using this probability measure we can derive the theoretical covariance in this Hilbert space of two current density distributions (equation 2). This is a measure of the overlap between two current density distributions. So, by fixing one current density distribution and mapping the covariance as outlined in this paper we can produce plots which indicate which sources are masked by the given source.

In Fig. 2 we see two such plots for dipolar sources (dipole are located at $(0,0.01 \mathrm{~m}, 0)$ and $(0.01 \mathrm{~m}, 0,0)$ resp., oriented along $x$ and $y$ resp.). This figure reproduces the pattern of insensitivity for a highly localised source which we might expect from previous experience with the biomagnetic inverse problem. 

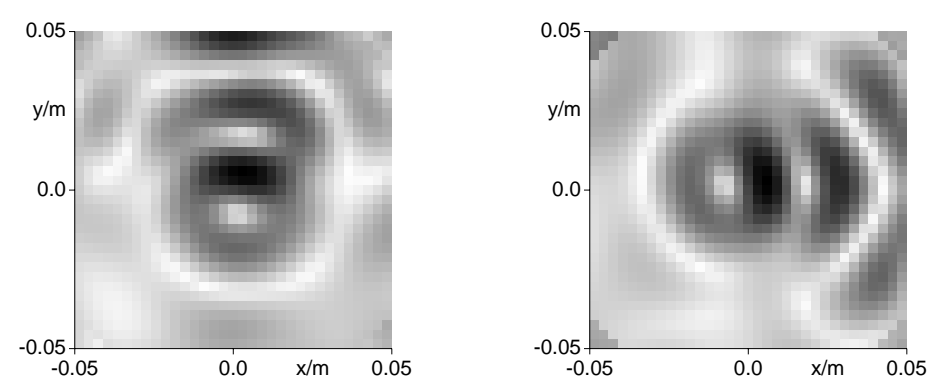

Fig. 2: (left) A dipole located at $(0.01 \mathrm{~m}, 0,0)$ oriented along $x$. Magnitude of the covariance plotted so that black represents insensitivity to a second source.

(right) A dipole at the same position but oriented along $y$.

More interesting perhaps is the covariance maps produced for multiple sources. These are shown in Fig 3 for two dipoles located at $(-0.05 \mathrm{~m}, 0,0)$ and $(0.05 \mathrm{~m}, 0,0)$ respectively. The three plots correspond to different relative orientations. Notice that the patterns are not simply a superposition of the covariance maps for the individual dipoles. Note also that the hexagonal symmetry of the measurement system is just visible.
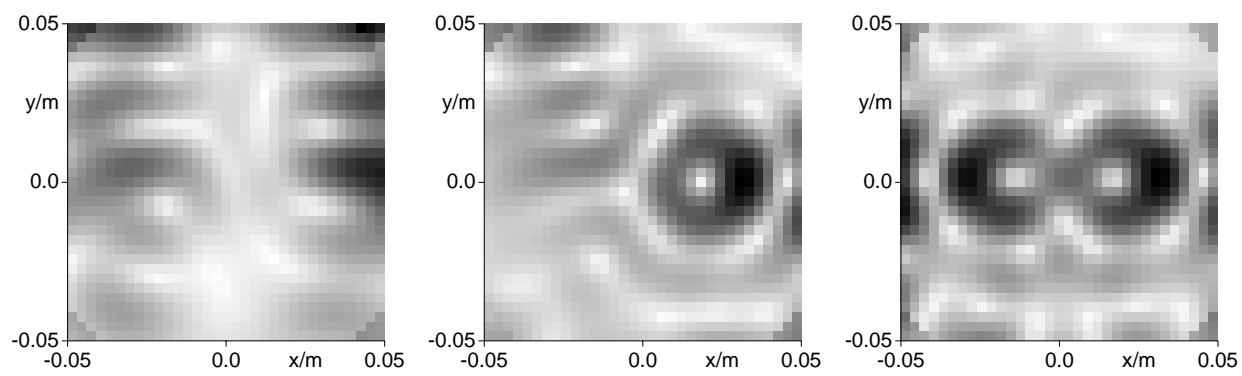

Fig. 3: (left) A dipole located at $(-0.05 \mathrm{~m}, 0,0)$ oriented along $x$ and another dipole located at $(0.05 \mathrm{~m}, 0,0)$ also oriented along $x$. Magnitude of the covariance plotted so that black represents insensitivity to a second source.

(middle) The same dipole positions but dipoles oriented along $x$ and $y$.

(right) The same dipole positions but dipoles oriented along $y$ and $y$.

Now, for the one dipole example, imagine rotating the source space so that the reconstruction plane is perpendicular to the plane of the gradiometers (this means that the top edge of the reconstruction plane is only $1 \mathrm{~cm}$ from the bottom coils of the gradiometers). In Fig. 4 the covariance maps are displayed for such a reconstruction plane. Note that because the reconstruction plane is so close to the gradiometers, the lead fields oscillate rapidly at the top of the reconstruction plane.

The main features to note in Fig. 4 are:

- The degree of masking is greater for sources in the reconstruction plane (Fig 4 (right)) than for sources perpendicular to the reconstruction plane (Fig 4 (left)).

- The contours of equal insensitivity form ellipsoids. This agrees with experience of the biomagnetic inverse problem: the resolution in the transverse plane is greater than the depth resolution.

- Contrary to intuition, a superficial source does not mask sources directly underneath it, provided the sources are sufficiently well separated. 

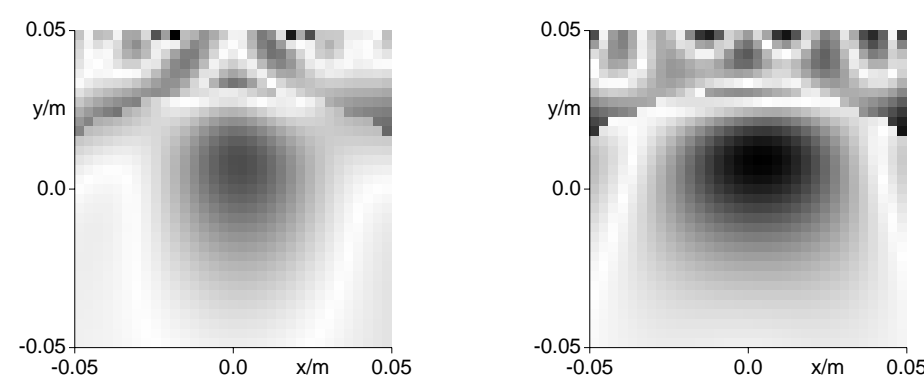

Fig. 4: (left) Depth profile of covariance for a dipole at the origin pointing perpendicular to the reconstruction plane.

(right) The same dipole position but with the dipole oriented in the plane of reconstruction.

We see this method as a means of evaluating whether a given instrument is capable of providing reliable information on a given postulated source distribution.

\section{References}

[1] Clarke, C.J.S., In: Nenonen, J., Rajala, H.-M., Katila, T. Biomagnetic localization and 3D modelling, Helsinki, TKK offset, 1991.

[2] Hasson, R., and Swithenby, S.J. In: Baumgartner, C., Deeke, L., Stroink, G., Williamson, S.J., BIOMAGNETISM: Fundamental research and clinical applications, 455-457, Amsterdam, Elsevier Science, 1995.

[3] Ioannides, A.A., Bolton, J.P.R., and Clarke, C.J.S. $\quad$ Inverse Problems, 1990, 6: 523-542. 